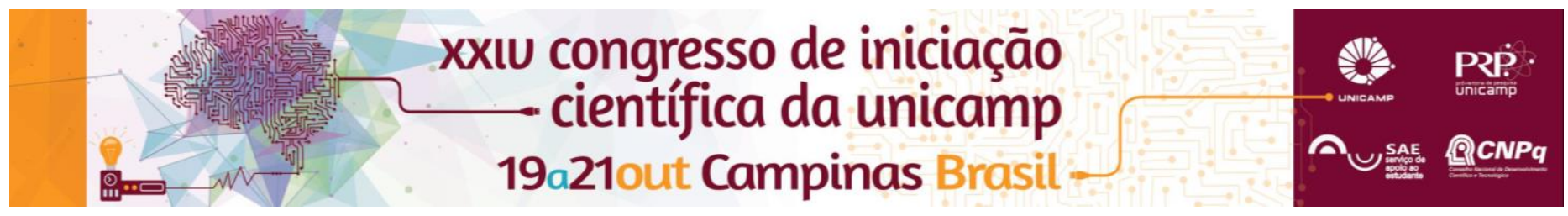

\title{
Amphiphilic Stackable Gels Synthesized by Atom Transfer Radical Polymerization
}

\author{
Rafael N. L. de Menezes*, Antoine Beziau, Hangjun Ding, Antonina Simakova, Tomasz Kowalewski, Maria I. \\ Felisberti, Krzysztof Matyjaszewski
}

\begin{abstract}
The synthesis of multilayered stackable gels based on 2-(dimethylamino)ethyl methacrylate and n-butyl methacrylate was performed using ATRP to combine layers with different properties. A pre-gel solution was introduced on top of a previously synthesized gel, and these new components then underwent ATRP to form the subsequent layer. In general it was shown that two layers sequentially synthesized by ATRP cannot be connected if immiscible solvents are used to synthesize each gel. It is believed that compositional and structural effects such as 'skin' layer effect are responsible for these observations. Successfull strategies to overcome these factors are presented.
\end{abstract}

\section{Key words:}

Atom Transfer Radical Polymerization (ATRP), Hydrogels, Multifunctional materials.

\section{Introduction}

Multi-functional materials address technological interest; however a challenge in the creation of these materials is the integration of different components into a single cohesive system. Recently, Matyjaszewski et.al studied multi-layered stackable gels. ATRP was used as a 'gluing' agent to effectively add one layer on top of the other through successive polymerization reactions. ATRP polymers are chain-end functionalized with halogens, as a consequence of the nature of reaction. Thus, the obtained polymers can be used as an initiator in a further ATRP reaction. This concept was used to prepare multilayered gels using the top of one gel as a source of ATRP initiator for the polymerization of the next layer (Figure 1). In this process, a solution of initiator, monomer, and cross-linkers was introduced on top of the first gel, and these components then underwent ATRP to form the subsequent layer. 2-(dimethylamino)ethyl methacrylate (DMAEMA) and n-butyl methacrylate (BMA) were used as monomers, and dimethylformamide (DMF) as a solvent. Multi-layered gels were obtained by using this approach. Herein, in order to test the limits of this method, immiscible solvents were used in each layer. Toluene was used to synthesize PBMA and water to synthesize PDMAEMA.

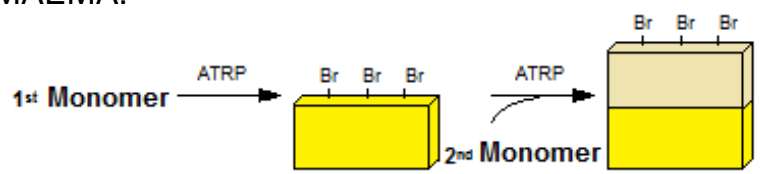

Figure 1. Representation of sequential stackable gel synthesis.

\section{Results and Discussion}

In general, layers sequentially synthesized by ATRP cannot be connected if immiscible solvents are used to synthesize each gel (Table 1), which is probably due to the poor interaction between the layers during the polymerization. Different approaches are employed in order to overcome this situation. Amphiphilic PBA-PEO mikto-arm stars were added prior the polymerization of the top layer, in order to enhance the interaction between the two layers during the polymerization of the second layer. This strategy resulted in a connection between the layers in Gel 4 (Table 1), which indicates that these mikto-arm stars may be used as compatilizers. Another approach to synthesize connected gels was the removal of the polymer/air interface of the bottom gel. Gels synthesized by ATRP are subject to a phenomenon socalled "skin layer" effect ${ }^{2}$, in which this polymer/air interface collapses and act as a barrier for transporting small molecules such as monomer and oligomers. As a consequence a decrease in the interaction between the layers during the polymerization of the top layer was observed. The comparison of Gels 6 and 3 proved that the skin effect seems to be of importance in obtaining stackable gels when immiscible solvents are used. However, only the gels in which PBMA was used as the bottom layer presented a strong connection. It addresses major differences in the structures and properties of the PDMAEMA gels comparison with the PBMA. Further studies are required to understand how these structural properties affect the synthesis of stackable gels in immiscible solvents.

Table 1. List of stackable gels synthesized. $(\checkmark)$ : connected gels and $(\mathrm{X})$ unconnected gels.

\begin{tabular}{cccc}
\hline Gel & Layers & Interface & $\begin{array}{c}\text { Conneceted } \\
\text { layers? } \\
\text { Bottom | Top }\end{array}$ \\
1 & PDMAEMA | PBMA & - & No \\
2 & PDMAEMA | PBMA & PEO-PBA mikto-arm star & No \\
3 & PBMA | PDMAEMA & - & No \\
4 & PBMA | PDMAEMA & PEO-PBA mikto-arm star & Yes \\
5 & PDMAEMA | PBMA * & Surface cut & No \\
6 & PBMA | PDMAEMA* & Surface cut & Yes \\
\hline
\end{tabular}

\section{Conclusions}

In general two layers could not be connected if immiscible solvents were used. Structural factors as 'skin' layer effect and the interaction between the layers during the polymerization of the second layer played a crucial role for obtaining stackable gels when immiscible solvents were used. The removal of the 'skin' layer and PEO-PBA miktoarm stars allowed the synthesis of connected gels.

\section{Acknowledgement}

FAPESP (process 2014/21392-7) and Carnegie Mellon University for the support and structure.

1 X Yong, A Simakova, S Averick, J Gutierrez, O Kuksenok, A Balazs, K Matyjaszewski, Macromolecules, 2015, 48, 116.

2 Y Kaneko, R Yoshida, K Sakai, Y Sakurai, T Okano, Journal of Membrane Science, 1995, 101, 13. 